\title{
Elementos para el desarrollo de las criminologías específicas desde la teoría de sistemas
}

Elements for the develop of the specific criminologies from the systems theory

DOI: $\underline{\text { https://doi.org/10.33262/rmc.v6i1.1083 }}$

\author{
Wael Sarwat Hikal Carreón ${ }^{1}$ \\ Universidad Autónoma de Nuevo León \\ (iD https://orcid.org/0000-0003-1278-567X \\ wael.hikalcrr@uanl.edu.mx
}

\section{RESUMEN}

Se presentan antecedentes de algunas criminologías específicas mencionadas por autores clásicos de la criminología, también tomados de referencia para escritores contemporáneos con propuestas de especialización en obras completas, capítulos en libro y eventos de divulgación. Posteriormente se reseñan las bases de la teoría general de los sistemas aplicada a la especialización de la criminología, señalando los propósitos concretos de esta vinculación. Como referentes de especialización se señalan las sociologías, psicologías y derechos específicos como salidas de investigación en las nuevas criminologías. Finalmente se presentan conceptos básicos de la teoría de los sistemas a manera de guía de desarrollo para términos, contenidos, enfoques y corpus teórico.

PALABRAS CLAVE: Abordaje de la criminalidad; Campos de conocimiento; Criminología; Especialización.

\begin{abstract}
Is presented a history of some specific criminologies mentioned by classical criminology authors, also taken as a reference for contemporary writers with specialization proposals in complete books, book chapters and events. Subsequently, the basis of the general theory of systems applied to the specialization of criminology is outlined, pointing out the specific purposes of this linkage. As references of specialization, sociologies, psychologies and specific laws are noted as research outlets in new criminologies. Finally, basic concepts of system theory are presented as a development guide for terms, content, approaches and theoretical corpus.
\end{abstract}

KEYWORDS: Addressing crime; Criminology; Fields of knowledge; Specialization.

\section{INTRODUCCIÓN}

En el nacimiento de los estudios de la criminología se han aglomerado una multitud de conocimientos que convergen en los ámbitos de lo criminal, el delito, la violencia, desviación, antisocialidad, psicopatía, sociopatía, entre otras, esto se convirtió luego en las llamadas "ciencias criminales", siendo la criminología parte de estas. Tales visiones sobre el fenómeno son tomadas por diversas ciencias (sociología, derecho, política, 
antropología, psicología, psiquiatría, demografía, estadística, entre otras), donde la criminología es la integradora de estos conocimientos para explicar el problema y aportar soluciones. "Pero la información no era suficiente para afrontar los cambios contemporáneos. Se hacía emergente el enfoque hacia una dimensión superior como la fuente principal para la solución ante la nueva situación: el conocimiento" (Nieves; Del Río; y Villardefranco, 2009, p. 3), por lo que aquí se propone que la criminología al igual que otras ciencias, madure y aporte sus propios saberes, explicaciones, teorías, términos y metodologías, ello a través de la especialización.

Los campos de especialización de la criminología pueden ramificarse en tantas áreas como las expresiones de la criminalidad; es decir, en aquellas situaciones donde pueda ocurrir un conato de crimen (y demás clasificaciones según las ciencias que convergen). La criminología puede especializarse para estudiar el fenómeno, descifrarlo, procurar explicaciones y propuestas de intervención a los planificadores de políticas públicas o privadas. El desarrollo de teorías propias a casos particulares, permitirá el crecimiento del corpus de la criminología, pero que dichas aportaciones se aglomeren en ramas especiales llamadas "criminologías específicas" en áreas como los medios de comunicación, lo digital, en lo social, familiar, escolar, de las causas, prevención, penitenciaria, femenil, entre otras. En las siguientes líneas se establecen las bases elementales para la creación de nuevas criminologías teniendo como referencia a las psicologías, sociologías y derechos específicos, así como las aportaciones que ya diferentes autores han llegado a las nuevas generaciones y que estas han realizado algunas contribuciones que también se muestran. Apunta Zaffaroni (1990) que: "pese a todas las dificultades, el interés por la investigación criminológica es creciente" (p. 69).

\section{Antecedentes de la especialización en criminología}

Señalaría Zaffaroni (1990): "por elementales razones de espacio renunciamos a detalladas referencias históricas (...), no por desconocer su incuestionable importancia (...) sino porque es casi imposible una síntesis que no pase por alto los matices que permiten su cabal comprensión" (p. 60). Por lo que se muestra una brevísima reseña de los desarrollado y observado en alrededor de 10 años.

Se han realizado algunos avances en la especialización de la criminología de manera formal, otros tal vez sin formalizarlo como línea de investigación, pero que han sentado conceptos y visiones, y alguno por mera novedad o marketing autopublicitario al tomar un tema de moda. En el primer rubro están los autores o conferencistas que desarrollan temas de especialización y se difunden en medios escritos (artículos o libros) o en conferencias. En el segundo aspecto, están autores que mencionaron la especialización en algún momento, pero sin dar continuidad, y en el último sentido, aquellos que lucran con los temas de novedad para publicitarse más que para aportar.

En los anales de la especialización de la criminología se encontró el libro de Reyes (2001) en el texto titulado Criminología, un libro tamaño tratado que menciona diversas criminologías específicas y se aportan conceptos de estas y algunos autores que se considera trabajaron sobre esa línea, por ejemplo, la criminología sociológica, y se encuentra a Sutherland, Cohen, Cressey, Cloward, entre otros (Hikal, 2019a). Otro referente ha sido la obra de Zaffaroni (2003), Criminología, quien menciona en diferentes 
momentos una serie de criminologías específicas, como la psicoanalítica, conductual, etiológica-multifactorial, sociológica, biológica, entre otras, y señala que los autores que construyeron el conocimiento de la criminología en sus inicios, pudiesen considerarse una inclinación hacía esos rubros.

Por otro lado, está Aniyar (2010) con su libro Criminología de los Derechos Humanos. Criminología Axiológica como Política Criminal. Volviendo con Zaffaroni (2012), este ha desarrollado la criminología mediática de manera textual en libros, así como un posgrado, Maestría en Comunicación y Criminología Mediática en la Universidad Nacional de la Plata, Argentina, en ambos se abordan temas de la construcción de la realidad basada en la información que difunden los medios de comunicación, creando panoramas de inseguridad, riesgo, etiquetamiento, etcétera.

De modo contemporáneo se encuentra la obra Introducción a la Criminología y a sus Metodología (2009) donde por primera vez en México, se presenta un capítulo dedicado a las criminologías específicas el cual se llamaba "La necesidad de reorganizar sistematizar el conocimiento criminológico", destacando las criminologías social, demográfica, ambiental, transcultural, familiar, educativa, de la consejería social, laboral, de la salud, del desarrollo, del arte, informática, biológica y filosofía criminológica, de tal manera, el nacimiento de las criminologías específicas en México se puede considerar el 13 de abril del 2009 cuando se publica dicho libro.

Derivado de este libro surgen iniciativas en otros títulos como Criminología EtiológicaMultifactorial (Hikal, 2015), Criminología de los Derechos Humanos (Hikal, 2016) (como segunda edición al libro titulado en la primera como Criminología, Derechos Humanos y Garantías Individuales, Hikal, 2013), Criminología Psicoanalítica, Conductual y del Desarrollo (Hikal, 2009), hoy titulada Criminología Psicológica (Hikal, 2019b), Criminología Sociológica (Hikal, 2019a). Obras de las cuales sus títulos derivaron de la lectura de Zaffaroni (2003) y Aniyar (2010).

Por otro lado, Criminología Clínica Contemporánea (Palacios, 2017), Criminologías Especializadas (Gómez, 2014), siendo esta una obra coordinada por el autor donde aglomera algunas criminologías en lo policial, de las migraciones, étnica, del deporte, de los derechos humanos, entre otras, pero lo ha tomado como un tema de moda sin darle continuidad al desarrollo, por lo que fungió para marketing personal. Criminología de Campo. Perfiles Criminales y Victimales (Echeverria, 2012), Criminología Reflexiva. Discusiones Acerca de la Criminalidad (Ordaz y Cunjama, 2011), igualmente, sin continuidad. Criminología Juvenil. Un Abordaje Multisciplinario (Hernández, Argüelles y Rangel, 2019).

En España se viene desarrollando la criminología vial, dedicada a la seguridad, se han impartido congresos, cursos, y propuestas de acción, surge también el libro Aspectos Criminológicos en Materia de Seguridad Vial (Carreras, 2014) lo cual representa un avance fuerte y de impacto en la especialización de la criminología y las estrategias de implementación en fenómenos concretos. En 2018, Ríos de Perú coordina la obra Criminologías Específicas, donde reúne a diversos autores con temas en criminología laboral, genética, de la religión, familiar, entre otros temas, siendo este el primer libro en aquel país. 
En otro enfoque, en México han surgido múltiples congresos, cursos y conferencias principalmente en criminología laboral o corporativa, preventiva, ambiental, ecológica, mediática, policial, escolar, femenil, así como de especialización. Por último, surgió una iniciativa llamada Colección de Criminologías Específicas (Hikal) donde a través de la recopilación de artículos de diversos autores de varios países se crearon libros en campos como la criminología de la violencia, criminología clínica, criminología de la personalidad antisocial, criminología penitenciaria, criminología preventiva, criminología conductual, entre otras.

\section{Teoría general de sistemas aplicada para la especialización de la criminología}

Según los autores Arnold y Osorio (1998) es una forma organizada y científica de construir la realidad desde una aproximación holística e integradora entre ciencias sociales y naturales; es decir desde múltiples disciplinas (propio de la criminología interdisciplinaria en sus comienzos). Esta teoría promueve un intercambio de información entre las diversas especialidades profesionales y científicas, y su conexión (por ello que se asemeje a un sistema integral).

Apunta Cisneros (2020): “Orden, desorden y organización conllevan cada uno el acomodo y la dinámica de condiciones y entes que, acomodados así y no de otra manera, dan forma a un abanico de posibles relaciones" (p. 63). Los objetivos de esta teoría aplicada en el proceso de especialización de la criminología, permitirá:

1. Impulsar el desarrollo de términos que reconozcan características y funciones de las nuevas criminologías (fronteras del conocimiento);

2. Desarrollo de teorías, leyes, metodologías y modelos aplicables a estos campos específicos de las criminologías (salidas de investigación);

3. Reducir la duplicidad y diversidad de explicaciones teóricas a través de la integración y desarrollo autónomo de conocimientos;

4. Promueve la creación, especialización, construcción de diferentes criminologías, a la vez, mantiene la unidad; es decir, relación entre el todo (criminología general) y sus partes (criminologías específicas), y

5. Establecer la formalización de su consolidación.

Hurwits indica: "la investigación criminológica todavía está (sic-está) en su infancia... no ha encontrado, y probablemente no encontrará nunca el "ábrete sésamo" de los misterios del mecanismo criminal, ni mucho menos la receta para una medicina eficaz que evite la futura criminalidad (como citó Reynoso, 2004, p. 7). Señalan Nieves; Del Río; y Villardefranco (2009) por tanto: "Gestionar el conocimiento posibilita la conversión del conocimiento tácito en explícito, permitiendo de esta forma que los conocimientos intrínsecos de cada individuo, sus habilidades, experiencias y competencias se compartan con los restantes (...)" (p. 3).

\section{Bases epistemológicas de las criminologías específicas}

En sus orígenes, la criminología nació principalmente de la antropología, sociología y psicología, así parece ser una cosmogonía del crimen, una visión y explicación desde diversos enfoques. Si se remontan a sus fuentes, quedaría como un conjunto de 
conocimientos religiosos, políticos, psicológicos, sociológicos y biológicos que se ocupan del crimen. Reynoso (2004) señala:

"Pero para mejor integración y justificación de la Criminología como ciencia autónoma, es necesaria la coordinación de los diversos conocimientos y reflexiones que se enfocan al crimen; pero como afirman Wolfgag y Ferracuti: "la historia de los esfuerzos hasta hoy promovidos para enlazar las ciencias de la conducta en una fructuosa cooperación no se ha visto premiada con éxitos impresionantes" (como citó Reynoso, 2004, pp. 3 y 4).

Con el tiempo, la antropología ha sido apartada de la criminología teniendo más injerencia el derecho. En las siguientes líneas se presentan tres de las ahora más importantes ciencias en relación a la especialización de la criminología. A esta variedad, se le llama: Interdisciplina, síntesis criminológica, ciencias criminológicas o ciencias penales. Estas áreas son: Derecho, psicología y sociología.

En el caso del primero, el delito ha sido regulado por el derecho; es decir, aparece una norma que describe, regula y controla determinada conducta, previo análisis sociológico, biológico, psicológico, inclusive religioso. Los seres humanos más que cualquier otra forma de vida requieren de una ley, ya que la vida de la sociedad es más precisa y delicada (Fregoso, 2017). La incidencia del derecho en la criminología es profunda, al grado de que el derecho penal establece las conductas que se van a investigar.

Por parte de la psicología para Kagan y Havemann (1972), la psicología "es la ciencia que estudia y trata de explicar la conducta observable y su relación con los procesos mentales que no se pueden ver y que suceden dentro del organismo, así como los eventos externos en el ambiente" (p. 9).

Respecto la última Gelles y Levine (1997) dan un concepto de sociología que se acerca en mucho a los efectos en materia criminal: "La Sociología es el estudio sistemático de los grupos y sociedades que construyen los humanos y de la forma en que estas relaciones afectan nuestra conducta” (p. 6). Gómezjara anota que la sociopatología:

Estudia las causas sociales de la conducta "desviada" (prostitución, delincuencia, minorías eróticas, vagancia) de sectores de la población, a partir de los valores y modelos considerados normales (leyes, tribunales) e impuestos (policía, ejército, penitenciarías, sanatorios psiquiátricos) a toda la sociedad por los detentadores del poder (p. 44).

En el caso de las dos anteriores permiten trabajar en la parte preventiva detectando conductas de riesgo proclives a ser delitos. Pero ¿En qué se puede tomar de referencia estas tres para la especialización en la criminología? En la siguiente tabla se muestra el desarrollo que han tenido, mismos campos en los que se puede desenvolver a la criminología mediante la conceptualización, investigación de campo, resolución de casos, construcción de la realidad, apropiación de fronteras de conocimiento, creación de metodologías, entre otras. 
Tabla 1

Cuadro comparativo

\begin{tabular}{|c|c|c|}
\hline $\begin{array}{l}\text { Sociologías } \\
\text { especializadas }\end{array}$ & Derecho especializado & $\begin{array}{l}\text { Psicologías } \\
\text { especializadas }\end{array}$ \\
\hline Teatro & Civil & Trabajo \\
\hline Zoosociología & Penal & Industrial \\
\hline Rural & Mercantil & Colores \\
\hline Radical & Ambiental & Militar \\
\hline Matemática & Electoral & Arte \\
\hline Marxista & Deporte & Humana \\
\hline Fenomenológica & Médico & Animal \\
\hline Crítica & Espacial & Social \\
\hline Comparada & Aeronáutico & Ingeniería \\
\hline Aplicada & Constitucional & Salud \\
\hline Trabajo & Tributario & Humanitaria \\
\hline Profesiones & Diplomático & Guerra \\
\hline Informática & Internacional & Emergencia \\
\hline Sociometría & Municipal & Forense \\
\hline Sociografia & Administrativo & Criminal \\
\hline Industria & Laboral & Clínica \\
\hline Desarrollo económico & Procesal & Anormal \\
\hline Consumo & Romano & Educativa \\
\hline Revolución & Canónico & Infanto-juvenil \\
\hline Partido político & Público & Fisiológica \\
\hline Imperialismo & Privado & Adolescencia \\
\hline Guerra & Militar & Alcoholismo \\
\hline Suicidio & Económico & Adicciones \\
\hline Mujer & Agrario & Videojuegos \\
\hline Religión & Informático & Desarrollo \\
\hline Fuerzas armadas & Sanitario & Conductual \\
\hline Fascismo & Urbanístico & Aplicada \\
\hline Enfermedades mentales & Bancario & Emociones \\
\hline Música & Corporativo & Deporte \\
\hline Medicina & Amparo & Sexo \\
\hline Moda & Fiscal & Marketing \\
\hline Inteligencia & Sucesorio & Consumidor \\
\hline Derecho & Comercio & Experimental \\
\hline Deporte & Humanos & Pareja \\
\hline Cultura & Seguridad Social & Familiar \\
\hline Conocimiento & Parlamentario & Ambiental \\
\hline Ciencia & Demográfico & \\
\hline Arte & Bioético & \\
\hline Empresa & Comparado & \\
\hline Ciudad & Marítimo & \\
\hline
\end{tabular}




\begin{tabular}{|ll}
\hline Educación & Indígena \\
Criminal & Social \\
Sexo & \\
Sindicato & \\
Lenguaje & \\
Familia & \\
\hline
\end{tabular}

Fuente: Hikal; Pérez Tolentino y Ramos Erosa (2019).

Zaffaroni (1990) señala que: "Es obvio que la investigación criminológica en América Latina no alcanza los niveles cualitativos ni cuantitativos de los países centrales, debido a los escasos recursos que se destinan a la misma y al limitado impulso oficial" (p. 69). De tal manera, el campo es amplio para trabajar y urgente al tener fenómenos criminales que evolucionan de manera rápida.

De las áreas vistas con anterioridad en las sociologías, derechos y psicologías, pueden aprovecharse el desarrollo que han tenido; por ejemplo, crear una criminología fenomenológica para la interpretación de la conducta y lo que le rodea, cómo se construye e interpreta esta (Hikal, 2020), o una criminología de la música para conocer los significados que los escuchas dan a ciertas letras musicales, ya sea por edades, géneros, vinculados al crimen, que fomenten el consumo de drogas, la promiscuidad, rebeldía, etcétera. Una criminología del trabajo, recordando la división en el trabajo de la que trataba Durkheim, por ejemplo, en la que existen desigualdades, diferencias, segregación de grupos, abuso laboral, condiciones inadecuadas, salarios desiguales.

La criminología se puede conectar con diversas áreas en las que las sociologías especializadas se han desarrollado, criminología comparada, criminología informática, criminología de las fuerzas armadas, criminología de la guerra, criminología de la religión, criminología de la mujer o femenil, criminología del deporte, criminología familiar, entre otras, donde en cada una hay expresiones de violencia, criminalidad o desviación. Pero tampoco puede conectar con todas; es decir, no cabría una criminología de la moda, o zoocriminología (o probablemente si la relación con los animales implicaría una conducta antisocial), el crecimiento en cada área dependerá de la capacidad de investigación de cada desarrollador.

Tomando de referencia a los derechos, no solo el derecho penal da un catálogo de delitos, sino las leyes especiales, por tanto, se puede tratar de una criminología jurídica penal permeando en áreas ambientales, espacial (criminología espacial), criminología laboral, criminología militar, criminología de los derechos humanos (donde la violación a estos constituye una conducta antisocial). En cada una habrá que revisar aspectos legales y criminales, incluso una criminología victimal. Respecto las psicologías se pueden derivar adaptaciones de modelos de estudio en criminología militar, criminología de los colores, criminología del arte, criminología educativa, criminología de las adicciones, criminología infanto juvenil, criminología del desarrollo, entre otras.

De nuevo se menciona a Zaffaroni (1990) cuando indica: "Entiendo que corresponde a la criminología una importantísima función en el futuro inmediato de América Latina, porque reúne el conocimiento (...) que es indispensable para hacer descender los niveles de violencia (...) (p. 70). 


\section{Elementos para la creación de las criminologías específicas}

El avance en el desarrollo de la criminología no representa un monopolio del conocimiento, pero sí una delimitación entre los campos y evitar dualidades o explicaciones laxas o incompletas. A continuación, se presentan algunos conceptos de la teoría general de sistemas (Arnold y Osorio, 1998) adaptados al contexto de la criminología para establecer un marco de referencia en la elaboración de las criminologías específicas; es decir un guía. Para evitar repeticiones por los diferentes abordajes al crimen desde la criminología interdisciplinaria (aportados por otras ciencias) como crimen, delito, sociopatía, psicopatía, violencia, etcétera, se generaliza con el término "crimen". Para construir una criminología específica podemos tener en cuenta los siguientes aspectos.

\section{Principio}

Basándose en la realidad de los escenarios donde se presenta el crimen, estudiados por cada ciencia que corresponde; por ejemplo, el crimen estudiado desde la sociología (o a futuro por la criminología sociológica), o desde una perspectiva psicológica clínica (o criminología clínica o criminología psicológica), quiere decir en totalidad, que cada ambiente donde el crimen se presente, corresponde a cierta ciencia y técnicas de estudio específicas. "Tiene tanta presencia como un elemento físico o un acto humano, (...) como algo que determina el modo de lo concreto y material"' (Cisneros, 2020, p. 78).

Cuando Zaffaroni (2003) titula a diferentes criminologías específicas, lo hace estableciendo fronteras de cada campo de ejercicio y desarrollo doctrinal y teórico; es decir, delimita cuando corresponde a una criminología biológica y los autores que en el ejercicio profesional de sus áreas, realizaban estudios entre lo médico, biológico, antropológico y lo criminal. O por otro lado, cuando menciona a la criminología psicoanalítica y los autores que pueden ser tomados de referencia, entre otras.

\section{Función y viabilidad}

¿Cuál sería la función de determinada criminología específica? Si pretendemos contribuir a la creación de esta especialización, se deberá visualizar cuál sería su alcance, utilidad, cuerpo de conocimientos que se tomen de otros campos que convergen y factibilidad. Se pone de ejemplo una criminología espacial (análisis de las conductas antisociales fuera de la Tierra) ¿Tiene lógica, soporte, alcances?

Si cada vez es más evidente la posibilidad de los viajes espaciales a través de las compañías Space X, Space Perspective, entre otras, así como las continuas observaciones y exploraciones a los planetas, la permanente misión de la Estación Espacial Internacional, etcétera, con ello vendrán conductas de disputa por dominio de territorios en otros planetas aunque en la actualidad toda exploración es con fines de beneficio para la humanidad, pero esto no demorará mucho en que exista la conquista por parte de algunos gobiernos, probablemente, del mismo modo en el que las naciones se fueron consolidando a través de conquistas, independencia, venta, donación, etcétera, puede ocurrir lo mismo en los planetas y lunas.

Lo anterior llevará a guerras, disputas, imposiciones, desafíos, esto por parte de los gobiernos, pero cabe pensar en la conducta humana que es tan cambiante ¿Qué pasará 
cuando ocurra el primer robo, homicidio, espionaje en otro planeta o luna? Tal vez de primera instancia le regulará la legislación nacional de donde provenga el individuo, pero cuando nazcan los primeros humanos fuera de la Tierra implicará cambios obligados a las leyes, quiere decir, nuevas formas de regulaciones, y con ello, nuevos crímenes, ahí cabe la criminología espacial... La viabilidad: "Indica una medida de la capacidad de sobrevivencia y adaptación" (Arnold y Osorio, 1998, p. 12).

Pero en un ejemplo más terrestre, se pueden enfocar los estudios en la criminalidad femenil, infanto juvenil, en los colores, medios de comunicación, laboral, del deporte, en cada uno (variedad) de estos contextos han surgido expresiones criminales que se abordan desde la psicología, sociología, antropología, derecho, estadística, estas ciencias podrán ser utilizadas como base teórica, doctrinal, epistemológica para la conversión a criminología, y con aquel conocimiento holístico e integrado se generará esa nueva criminología especializada en alguna de esos campos.

\section{Ambiente}

Se refiere al lugar donde ocurren los crímenes y las condiciones que influyen, la complejidad, los ambientes sociales donde ocurre el crimen son diferentes, cambiantes, o estables, con condiciones particulares que denotan la modalidad criminal, no es lo mismo un robo en banco que en tienda de abarrotes, tampoco el homicidio a un usuario del transporte público que a un servidor público de alto nivel. Cada ambiente hace particular a la forma de expresión del crimen, tiene propiedades estructurales y funcionales.

\section{Circularidad}

Quiere decir la dinámica entre las causas criminógenas y factores criminógenos, cómo los procesos influyen unos en otros. Por ejemplo, si el ambiente es criminógeno, cómo esto influye en la constitución y desarrollo de determinadas formas criminales.

\section{Conglomerado}

Es el estudio y detección de todo aquello presente en el ambiente, y su interacción, de tal forma, son los elementos o partes que lo constituyen, cómo se organizan o desorganizan (involucramiento de autoridades en el crimen, de la misma población).

\section{Equifinalidad}

Tomando los términos anteriores se puede observar que la finalidad en algunos ambientes incita al crimen; es decir, las condiciones se aglutinan para provocar el crimen donde todos sus elementos funcionan en torno al crimen y para el crimen. Ejemplo de ello está en las comunidades donde a los líderes del crimen organizado tienen más respeto e idolatría que otras figuras legales; es decir, un capo puede ser visto como héroe, mientras que un presidente como un tirano.

\section{Emergencia}

"La emergencia se entiende como la aparición inesperada de una nueva realidad, de un objeto o ente que se cocina en el interior (...)" (Cisneros, 2020, p. 71). En el caso de la criminalidad, emergen conductas previsibles en ocasiones, pero otras que toman por 
sorpresa a los profesionales y científicos; por ejemplo, vacunas falsas de COVID, explosión de ductos de gasolina, rapiña cuando se volca un camión de transporte de mercancía, desaparición de menores, entre tantos hechos.

\section{Frontera}

Si se fragmentan las zonas de crimen, cada una posee los elementos anteriores, aquí podría nacer una criminología transcultural o criminología comparada, donde establece diferencias entre las formas de expresión de la criminalidad. Puede haber zonas donde los crímenes violentos en transporte público sean una constante, mientras que, en otras, las riñas, venta de drogas, otras con fraudes bancarios, compra de facturas falsas, etcétera, cada región tiene fronteras criminales en los modos de operar.

\section{Información}

Se deberá acumular contenidos de información referentes a algo previo que exista sobre el fenómeno que se está investigando (bases tomadas desde la demografía, estadística, derecho, antropología, sociología, psicología, etcétera), o de no existir fuentes, desarrollarlas, en esto nace la autonomía de las nuevas criminologías, primero como acumuladoras y sistematizadoras de información relacionada al objeto específico (por ejemplo, criminalidad de menores o conductas adictivas y crimen), para luego integrar y crear nuevas propuestas (morfogénesis, diversidad de formas, estructuras, estado de especialización, aprendizaje). Ello dará solidez a los conocimientos que aporta la criminología con un listado de explicaciones, propuestas, soluciones, cambios.

\section{Input y output}

El input se refiere a la importancia de los recursos informativos con los que se cuenta y dar comienzo a la elaboración de la criminología específica. Mientras que el output a las salidas que esa información tendrá (utilidad de la criminología según funciones, servicios, soluciones).

\section{Organización}

Al organizar a las diferentes criminologías específicas creadas producirá una nueva identidad en cada una de estas y a la vez diversidad que se requiere para efectivamente complementarse unas de otras. "Mientras más interacciones entre contrarios y complementos se den, más complejidad encontrará la organización y más autónoma será” (Cisneros, 2020, p. 75).

\section{Relación y retroalimentación}

Serán los efectos recíprocos entre todos los elementos anteriores, el principio relacionado con la realidad de los escenarios donde existen las expresiones criminales. La función de una criminología específica focalizada a ciertos fenómenos (organización y frontera). El ambiente donde ocurre el crimen y sus características. La circularidad por la relación de factores criminógenos en la influencia de las diferentes conductas. La conglomeración de partes que constituyen el crimen y a la misma criminología específica, su organización (utilidad de la información). La equifinalidad en los ambientes criminógenos, pero 
también de la misma criminología, su fin último es el explicar, resolver, proponer (con la información input y output). La emergencia por la explicación de los fenómenos que surgen de modo espontáneo, al final de la organización se tienen los elementos unidos.

\section{CONCLUSIONES}

Se presentó una guía para el desarrollo de las criminologías específicas basándose en la teoría general de los sistemas, comprende una metodología de construcción, donde los elementos importantes son: a) la observación de la conducta criminal en ambientes, b) identificación de su estructura, c) bases doctrinales y teóricas de donde se puedan sostener estas nuevas criminologías y sus explicaciones a los fenómenos, d) determinar atributos, utilidad, relaciones, y e) finalmente la organización y unión del todo. El seguir esta guía no implica que se esté libre de errores, cambios, agregar otros elementos, descartar algunos o basarse en otras estructuras, la consolidación de la especialización de la criminología no es un proceso sencillo, está en sus primeras etapas, pero siguiendo lo que otros autores han desarrollado, así como en otros continentes, se puede tener ruta para imitar. La comprobación de la utilidad de las criminologías nuevas o específicas se determinará en la medida que cumplan con los objetivos sobre las cuales fueron creadas.

\section{REFERENCIAS BIBLIOGRÁFICAS}

Aniyar, L. (2010). Criminología de los Derechos Humanos. Criminología Axiológica como Política Criminal. Ediciones del Puerto.

Arnold, M. y Osorio, F. (1998). Introducción a los conceptos básicos de la teoría general de sistemas. Cinta de Moebio. (3), 1-11. https://www.redalyc.org/pdf/101/10100306.pdf

Carreras, J.A. (2014) (coord.). Aspectos Criminológicos en Materia de Seguridad Vial. Criminología y Justicia Editorial.

Cisneros, J.L. (2020). Filosofía del Pensamiento Complejo. Una Reflexión sobre Edgar Morín. Centro de Estudios Humanísticos. https://libros.uanl.mx/index.php/u/catalog/download/66/78/221-1 ?inline=1

Echeverria, F. (2012). Criminología de Campo. Perfiles Criminales y Victimales. Trillas. Fregoso, J. (2017). El derecho en la cultura. Revista Misión Jurídica, 10(13), 109-135. https://www.revistamisionjuridica.com/wp-content/uploads/2020/09/4-Elderecho-en-la-cultura.pdf

Gelles, R. y Levine, A. (1997). Introducción a la Sociología. McGraw-Hill.

Gómezjarra, F.A. (2008). Sociología. Editorial Porrúa.

Gómez, E. (2014) (coord.) Criminologías Especializadas. Asesoría de Diseños Normativos.

Hernández, C., Argüelles, E.E. y Rangel, X.G. (2019). Criminología Juvenil. Un Abordaje Multisciplinario. Flores Editor y Distribuidor.

Hikal, W.S. (2020). Interpretación del otro como criminal. Bases para la construcción de una criminología fenomenológica. Ratio Juris, 15(30), 174-185. https://publicaciones.unaula.edu.co/index.php/ratiojuris/article/view/771/1044

Hikal, W. (2019a). Criminología Sociológica. Teorías Sociológicas de la Criminalidad. Flores Editor y Distribuidor. 
Hikal, W. (2019b). Criminología Psicológica. Teorías Psicológicas de la Criminalidad. Porrúa.

Hikal, W.; Pérez Tolentino, J.A., y Ramos Erosa, R.A. (2019). Nacimiento, sistematización y evolución de las criminologías específicas en México. Derecho $\quad y \quad$ Cambio Social. $\quad$ 57, 368-408. https://lnx.derechoycambiosocial.com/ojs-3.1.14/index.php/derechoycambiosocial/article/view/171/102

Hikal, W. (2016). Criminología de los Derechos Humanos. Porrúa.

Hikal, W. (2015). Criminología Etiológica-Muiltifactorial. Los Factores Criminógenos. Flores Editor y Distribuidor.

Hikal, W. (2013). Criminología, Derechos Humanos y Garantías Individuales. Porrúa. Hikal, W. (2009a). Introducción al Estudio de la Criminología y a su Metodología. Editorial Porrúa.

Hikal, W. (2009b). Criminología Psicoanalítica, Conductual y del Desarrollo. Flores Editor y Distribuidor.

Kagan, J. \& Havemann, E. (1972). Psychology. New York: Harcourt Brace Jovanovich Inc.

Nieves, Y.; Del Río, Y.; y Villardefranco, M.C. (2009). Elementos esenciales para la identificación del conocimiento organizacional en especialidades universitarias cubanas. Ciencias de la Información, 40(2), pp. 3-13. https://www.researchgate.net/profile/YadiraNieves/publication/258265606_Elementos_esenciales_para_la_identificacion_ del_conocimiento_organizacional_en_especialidades_universitarias_cubanas/li nks/5dab3f284585155e27f6f086/Elementos-esenciales-para-la-identificaciondel-conocimiento-organizacional-en-especialidades-universitarias-cubanas.pdf

Ordaz, D. y Cunjama, E.D. (2011). Criminología Reflexiva. Discusiones Acerca de la Criminalidad. Ubijus.

Palacios, G.S. (2017). Criminología Clínica Contemporánea. Porrúa.

Reyes, J.A. (2001). Criminología. Cárdenas Editor Distribuidor.

Reynoso, R. (2004). Nociones de Criminología e Historia del Derecho Penal. Cárdenas Editor y Distribuidor.

Ríos, G. (2018) (coord.). Criminologías Específicas. Instituto Pacífico.

Zaffaroni, E.R. (2012). La Cuestión Criminal. Planeta.

Zaffaroni (2003). Criminología. Aproximación desde un margen. Temis.

Zaffaroni (1990). La enseñanza universitaria de la Criminología en América Latina.

PARA CITAR EL ARTÍCULO INDEXADO.

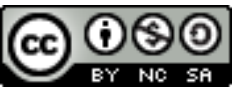

Eguzkilore. Cuaderno del Instituto Vasco de Criminología. 3. https://www.ehu.eus/documents/1736829/2164896/08++ La+ensenanza+universitaria+de+la+criminologia.pdf 